\title{
Growth and Characterization of Titanium Doped Spinel Crystals
}

\author{
V.T. Gritsyna ${ }^{a, *}$, Yu.G. KAZARINOV ${ }^{a, b}$, V.A. Kobyakov ${ }^{a}$ And L.A. LytVynov ${ }^{c}$ \\ ${ }^{a}$ V.N. Karazin Kharkiv National University, 4, Svoboda Sq., Kharkiv, 61022, Ukraine \\ ${ }^{b}$ NSC Kharkov Institute of Physics and Technology, 1, Akademicheskaya Str., Kharkiv, 61108, Ukraine \\ ${ }^{c}$ Institute for Single Crystals of NAS of Ukraine, 60 Nauky Ave., Kharkiv, 61001, Ukraine
}

\begin{abstract}
$\mathrm{MgAl}_{2} \mathrm{O}_{4}$ (magnesium aluminates spinel, MAS) single crystals doped with titanium ions obtained by the Verneuil method were non-uniformly colored along of the growth crystallographic direction. The crystals were characterized measuring lattice parameter, optical absorption spectra and relative concentration of impurity atoms $(\mathrm{Ti}, \mathrm{Mn}, \mathrm{Cr}$, and $\mathrm{Fe}$ ) in different spots of grown Ti:MAS boule. There was observed the correlation of the intensity of absorption band at $800 \mathrm{~nm}$ to the relative concentration of iron supporting the identification of this band with charge transfer transition in complexes $\mathrm{Ti}^{4+}+\mathrm{Fe}^{2+} \rightarrow \mathrm{Ti}^{3+}+\mathrm{Fe}^{3+}$. The new resonant structure at the wavelength of $470 \mathrm{~nm}$ was found and tentatively identified with the Fano resonance in the complexes formed by lattice defects and impurity ions.
\end{abstract}

DOI: 10.12693/APhysPolA.133.774

PACS/topics: $81.10 . \mathrm{Fq}, 61.10 . \mathrm{Nz}, 42.70 . \mathrm{Hj}$

\section{Introduction}

Magnesium aluminates spinel (MAS) $\mathrm{MgAl}_{2} \mathrm{O}_{4}$ possesses a number of very important properties such as high melting point, low thermal expansion, radiation and chemical resistance, and wide range of the optical transparency. These features of MAS make it as potential candidate for a variety of technological applications, particularly the transition metals doped spinel are recommended for high temperature fiber optic thermometer [1], high intensity white light source [2], etc. Recently there appears the interest for growth and investigation of the properties of titanium doped magnesium aluminates spinel (Ti:MAS) crystals for possible laser application and white emission phosphor [3-5].

It turned out that the optical properties of grown crystals are dependent on the growth conditions. Grown by the Verneuil method Ti:MAS crystals demonstrates absorption bands at 790, $490 \mathrm{~nm}$ and strong absorption in UV range [3]. In absorption spectra of the crystals grown by micro-pulling-down ( $\mu$-PD) method Ti:MAS of the different titanium concentration also three absorption bands were observed [4]. But crystals grown using the floating zone method under oxidizing atmosphere have no absorption bands except one in the UV-range [5]. The luminescence properties of grown crystals are also different. For the $\mu$-PD grown crystals the excitation into UV absorption region produces a broad emission band at wavelength of $455 \mathrm{~nm}$ [6], but in crystals grown be floating zone methods the strong blue emission band shifts to $490 \mathrm{~nm}$ [7]. For the phosphor powder samples of Ti:MAS there was observed white emission peak consisting of sev-

*corresponding author; e-mail: vtgritsyna@karazin.ua eral peaks when was excited by $260 \mathrm{~nm}$ wavelength [8]. It was shown that both absorption and emission bands in blue range of wavelength are highly overlapped [9].

In this paper we have grown MAS crystals doped with titanium at concentration of 0.2 mass\% using the Verneuil method which were non-uniformly colored in bluish. The optical properties and relative concentration of titanium and the impurity atoms of differently colored spots were measured to disclose the origin of absorption centers formation. In the course of this investigation we found well exhibited resonant absorption which tentatively was assigned to the Fano resonance and its transformation at different treatment was investigated.

\section{Experimental}

The crystals sizes of $30-40 \mathrm{~mm}$ in length and about $20 \mathrm{~mm}$ in diameter were obtained at growth rate about $20 \mathrm{~mm}$ per hour. The measured lattice parameter equals to $a=0.7996 \pm 0.0004 \mathrm{~nm}$ which corresponds to crystal lattice of non-stoichiometric spinel $\mathrm{MgO} \cdot 2.5 \mathrm{Al}_{2} \mathrm{O}_{3}$. No variation in spinel composition across the boule was registered. Some of the samples cut from boule were checked on the composition by means of X-ray photoelectron spectroscopy (XPS) using method developed in [10] and confirmed indicated composition of samples.

Optical absorption spectra of Ti:MAS crystals have been investigated in order to find out the nature of bluish coloration in obtained crystal. The single crystals were cut along of growth direction in slices of $2.0 \mathrm{~mm}$ of thickness and polished to optical finish. As can be seen in Fig. 1 (inserted picture) crystal was colored nonuniformly in blue along the length of boule. The absorption spectra were measured using single beam spectrophotometer SF-2000 in spectral region of 190-1100 nm in different colored and uncolored spots of samples. For 
the thorough measurements of absorption in chosen spectral interval we used spectrophotometer SF-16. At the same spots the relative concentration of impurity ions of Ti, Cr, Mn, and Fe was measured using X-ray fluorescent analyzer KRAB-3UM.

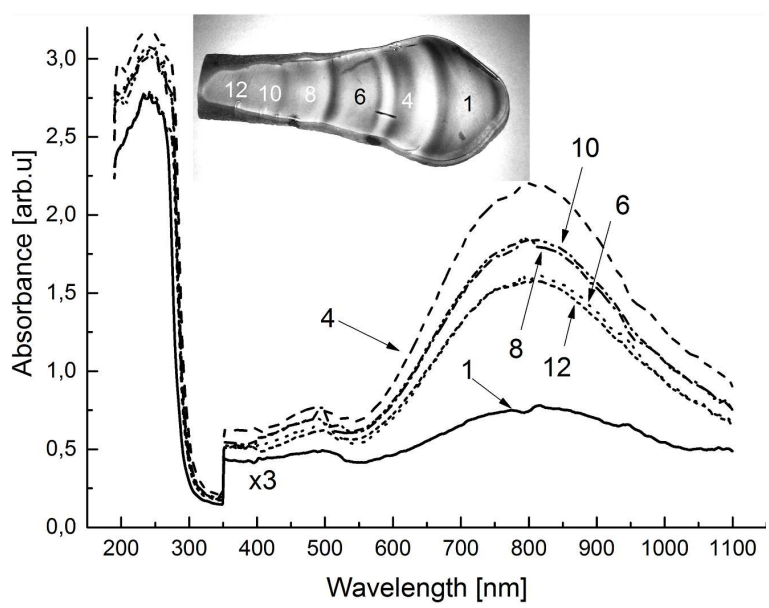

Fig. 1. Cross-section view of Ti:MAS crystals doped with titanium at concentration of 0.2 mass\% and absorption spectra measured in indicated spots.

Absorption spectra were measured for as grown samples and irradiated with UV-light or X-rays. For UVirradiation we used deuterium LDD-400 lamp of the emission spectral range $160-420 \mathrm{~nm}$. X-ray irradiation was provided using X-ray source URS-55 and $\mathrm{Cu}$ X-ray tube BSW-2 working at the voltage of $40 \mathrm{kV}$ and current $10 \mathrm{~mA}$. Irradiated samples were annealed in quartz pipe open to air to maximal temperature of $650{ }^{\circ} \mathrm{C}$ during $20 \mathrm{~min}$. Cooling of samples to room temperature was together with quartz pipe.

\section{Results and discussion}

\subsection{Variation of the optical absorption centers in Ti:MAS}

The absorption spectra of spinel crystals doped with titanium contain strong and wide band of maximum at $800 \mathrm{~nm}$, the intensity of this band measured in colored spots is higher to compare with that of colorless ones (Fig. 1).

Another low intensity asymmetrical band at $480 \mathrm{~nm}$ was also observed, the intensity of which also increased in colored spots. In the UV range a strong absorption edge arising from $300 \mathrm{~nm}$ is observed and red shift of this edge correlates to the intensity of the $800 \mathrm{~nm}$ band which is shown in Fig. 2.

In different spots of the single crystal where the absorption spectra were measured we also attained the relative concentration of impurity atoms. Therefore, we obtained the distribution of impurity ions along of the growth direction of crystal (Fig. 3). As can be seen from this picture no noticeable variation of titanium atoms concentration measured by X-ray fluorescent analysis was

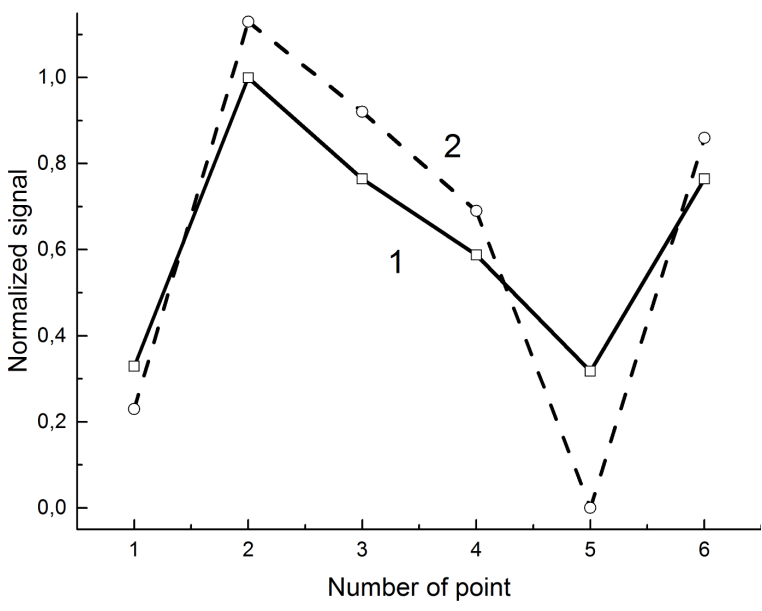

Fig. 2. Correlation of normalized optical density in the maximum of the absorption band at $800 \mathrm{~nm}$ (1) to normalized shift of absorption edge at $300 \mathrm{~nm}$ (2) for different spots of spinel single crystals.

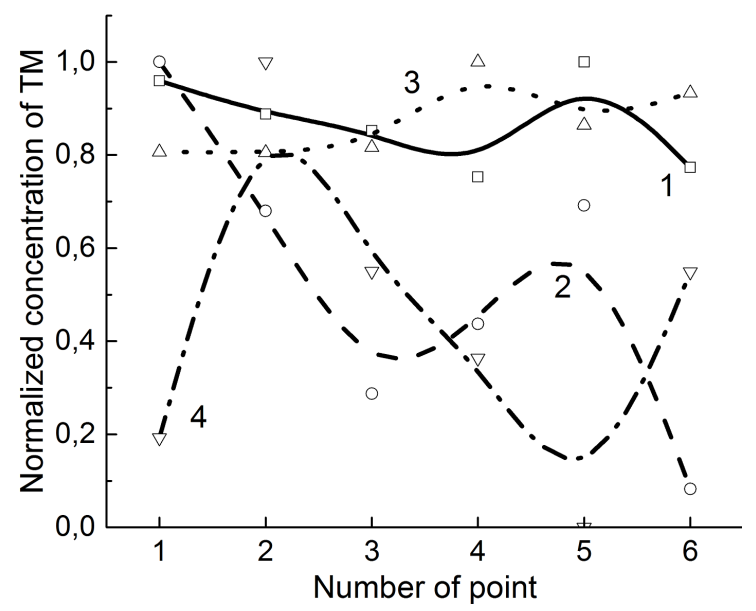

Fig. 3. Distribution of normalized concentration of different metal impurities along of the length of spinel single crystals Ti:MAS: $1-\mathrm{Mn}, 2-\mathrm{Cr}, 3-\mathrm{Ti}, 4-$ Fe.

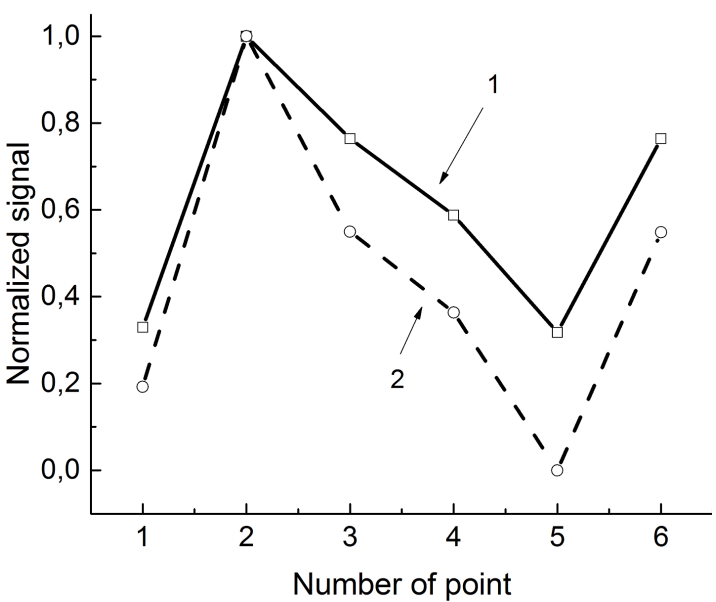

Fig. 4. Correlation of normalized optical density of band at $800 \mathrm{~nm}$ (1) to normalized concentration of iron atoms (2) in different spots of single crystals Ti:MAS. 
observed. Also we conclude that concentration of the manganese atoms was not changed in the limit of experimental errors. The comparison of variation of relative concentration along crystal for impurity iron shows that the intensity of optical absorption band at $800 \mathrm{~nm}$ is correlated to the content of iron atoms (Fig. 4).

From the analysis of the obtained experimental data we conclude that absorption band at $800 \mathrm{~nm}$ is related to complexes of the iron and titanium ions in corresponding charged (valence) states [3]. The identification of this band with charge transfer transition between coupled iron-titanium ions pairs $\mathrm{Ti}^{4+}+\mathrm{Fe}^{2+} \rightarrow \mathrm{Ti}^{3+}+\mathrm{Fe}^{3+}$ was supported by other publications [6]. The origin of the variation of iron concentration along the growth direction is not clear because the spinel powder was not changed, but gas composition of flame can be changed accidentally to some degree leading to oxidizing or reducing potential of the growth atmosphere. This may influence on the charge states of impurity ions participating in forming of complexes.

The spectral position of absorption band at $480 \mathrm{~nm}$ very good conforms to the transition $t_{2 g}^{1} \rightarrow e_{g}$ in the $\mathrm{Ti}^{3+}$ ions in octahedral sites. The variation of the intensity of this absorption band in colored and colorless spots additionally indicates on the change of composition of growth atmosphere. The absence of absorption bands at 480 and $800 \mathrm{~nm}$ in crystals grown by the floating-zone method in an oxidizing atmosphere supports in some way the role of growth atmosphere [5]. Also these crystals were the stoichiometric ones in composition whereas others (including ours) were non-stoichiometric crystals.

The absorption in UV range is characterized by strong absorption edge arising from $300 \mathrm{~nm}$ and peaking at $230 \mathrm{~nm}$ demonstrates red-shift of the absorption edge in dependence on the titanium concentration [6, 11]. Our results show the correlation of this shift to intensity of the $800 \mathrm{~nm}$ absorption band at the invariable titanium concentration. The detailed measurements of absorption in UV range indicate the existence of the regular poorly resolved peaks at wavelength of $260,253,244$, and $233 \mathrm{~nm}$. The correlation of the intensity of $800 \mathrm{~nm}$ band to value of shift of the absorption edge at $300 \mathrm{~nm}$ indicates the common nature of these two types absorptions, at least, pairs of $\mathrm{Ti}^{4+}+\mathrm{Fe}^{2+}$ participate in formation of the charge transfer transitions in the UV absorption region. The relative intensity of these bands changes after irradiation with UV-light or X-rays but now data is not enough to identify species responsible for these absorption bands.

\subsection{Origin of the Fano resonance in Ti:MAS}

In as grown spinel crystals we found some irregularities in measured absorption at the region of wavelength 470 and $790 \mathrm{~nm}$ (Fig. 5). The precise measurements of absorption in these wavelength ranges show the existence of rather intense non-symmetrical peaks which presumably were attributed to impurity complexes and formation of sub-bands in the band of forbidden states in crystal lattice and, as consequence, to appearance of the Fano resonance. The most prominent evidence on the presence of resonant feature is seen in spectra measured in the bluish colored spots. The similar resonance was observed in sapphire doped with titanium and iron grown under oxidizing conditions by the Verneuil methods at the same wavelength $480 \mathrm{~nm}$ and the same shape of peak [12]. There is some other indication on the existence of the irregularities in dependence of absorption on the wavelength of the incident light beam, particularly, in the vicinity of $800 \mathrm{~nm}$ (Fig. 5).

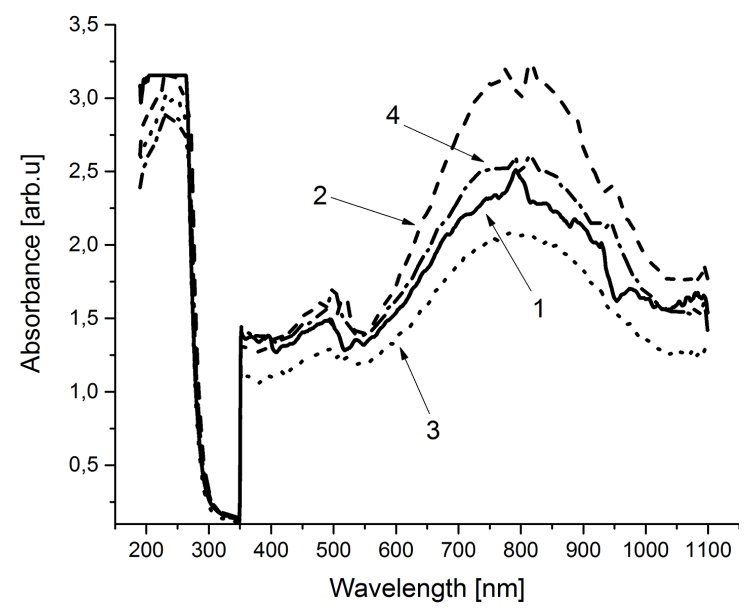

Fig. 5. Absorption spectra measured in colored places $(1,2)$ and colorless places $(3,4)$ in as grown Ti:MAS single crystals.

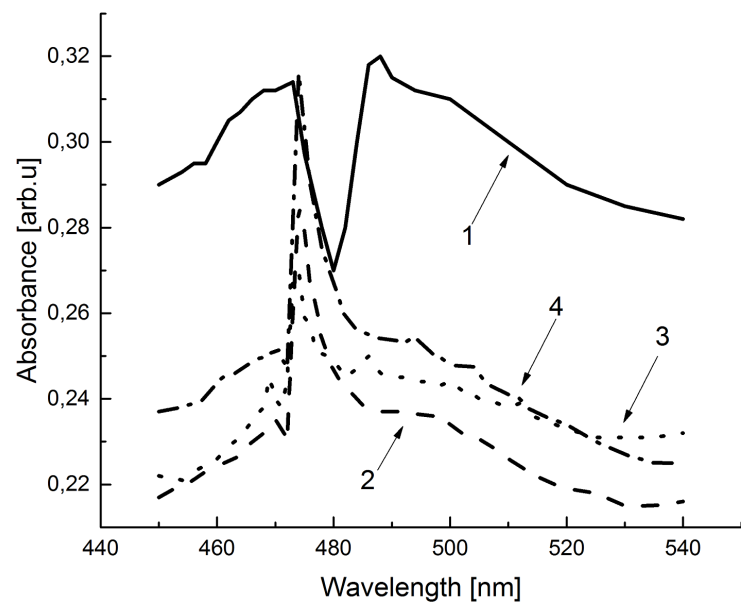

Fig. 6. Absorption spectra in the range of wavelength 450-540 $\mathrm{nm}$ measured in the Ti:MAS single crystals (colored spot): 1 - as grown, 2 - annealed to temperature of $650{ }^{\circ} \mathrm{C}, 3$ - irradiated by UV-light for $15 \mathrm{~min}$, 4 - irradiated with X-rays for 15 min.

The detailed absorption spectra in the wavelength range of 450-540 $\mathrm{nm}$ after different treatment are shown in Fig. 6. In the as grown crystals in the vicinity of the wide absorption band related to $\mathrm{Ti}^{3+}$ ions which are situated in octahedral sites there appeared damping of absorption in the form of narrow antiresonance of minimal absorption at $479.8 \mathrm{~nm}$. At the annealing to temper- 
ature of $650^{\circ} \mathrm{C}$ it changes to the resonance peaking at $474.3 \mathrm{~nm}$. Absorption band due to transitions in $\mathrm{Ti}^{3+}$ ions also considerably decreased. The irradiation with UV light does not change the spectral position and intensity of resonance; also, there appear satellites at 469.0 and $486.0 \mathrm{~nm}$. Finally, irradiation with X-rays causes increase of absorption band due to the transition in $\mathrm{Ti}^{3+}$ ions. The last phenomenon is understandable because Xray ionization creates free electrons which are captured by isolated $\mathrm{Ti}^{4+}$ ions increasing the growth of number $\mathrm{Ti}^{3+}$ ions. Therefore, the observed in optical absorption spectra the band at $470 \mathrm{~nm}$ in Ti:MAS single crystals indicated the existence of the $\mathrm{Ti}^{3+}$ isolated ions which after annealing in air to $650{ }^{\circ} \mathrm{C}$ reduced in intensity and undergo some rearrangement in structure that leads to appearing of the Fano resonance instead of anti-resonance.

It should be noted that in nominally pure spinel of different composition there are large concentration of point charged defects that are formed at the interchange of cations between oxygen tetra- and octahedral sites, so called anti-site defects (i.e. $\mathrm{Mg}^{2+}$ ions in octahedral sites forms $\left[\mathrm{Mg}_{\mathrm{Al}}^{2+}\right]$ defect, $\mathrm{Al}^{3+}$ ions in tetrahedral sites forms $\left(\mathrm{Al}_{\mathrm{Mg}}^{3+}\right)^{+}$defects). As it was shown before some of these defects are spatially correlated [13]. In the nonstoichiometric spinel $\left(\mathrm{MgO} \cdot n \mathrm{Al}_{2} \mathrm{O}_{3}\right)$ of $n>1.0$ the excess $\mathrm{Al}^{3+}$ ions are located in tetrahedral sites and for charge compensation the additional cationic vacancies are formed [14]. The recent calculation of the native defects in MAS shows the preferential formation of complexes of defects $\left(\mathrm{V}_{\mathrm{O}}+\mathrm{O}_{i}\right),\left(\mathrm{V}_{\mathrm{O}}+\mathrm{Al}_{\mathrm{Mg}}\right),\left(\mathrm{V}_{\mathrm{O}}+\mathrm{Mg}_{\mathrm{Al}}\right)$, and $\left(\mathrm{Mg}_{\mathrm{Al}}+\mathrm{Al}_{\mathrm{Mg}}\right)$ which form energy levels in band gap of spinel [15]. These complexes at different charge states may serve as centers for attraction of impurity ions $\mathrm{Ti}$ and Fe forming nanosized plasmon complexes. The investigation of cathodoluminescence in UV range of emission in doped nano-alumina also demonstrates the Fano resonance in ionized Cr-vacancy complexes [16].

\section{Conclusions}

The magnesium aluminates spinel crystals doped with titanium ions were grown using the Verneuil method. At the chosen growth conditions we obtained Ti:MAS crystals of the macroscopic strip colored in bluish along growth direction. The measurements of optical absorption spectra and concentration of impurity ions in differently colored strips allow us to identify the absorption band at $800 \mathrm{~nm}$ to charge transfer transition between coupled iron-titanium ions pairs $\mathrm{Ti}^{4+}+\mathrm{Fe}^{2+} \rightarrow \mathrm{Ti}^{3+}+\mathrm{Fe}^{3+}$, the band at $470 \mathrm{~nm}$ to transition in $\mathrm{Ti}^{3+}$ ions in octahedral coordination and absorption in UV range to charge transfer transition in complexes of defects and impurity ions.
These complexes create energy levels in the band gap of spinel crystals that stimulate the appearance of the Fano resonance. By different treatments (irradiation and annealing) we can change the intensity of this resonance.

\section{References}

[1] H. Aizawa, N. Ohishi, S. Ogawa, E. Watanabe, T. Katsumata, S. Komuro, T. Morikawa, E. Toba, Rev. Sci. Instrum. 73, 3089 (2002).

[2] Y. Fujimoto, H. Tanno, K. Izumi, S. Yoshida, S. Miyazaki, M. Shirai, K. Tanaka, Y. Kawabe, E. Hanamura, J. Lumin. 128, 282 (2008).

[3] L.E. Bausa, I. Vergara, J. Garsia-Solé, W. Strek, P.J. Deren, J. Appl. Phys. 68, 736 (1990).

[4] A. Jouini, H. Sato, A. Yoshikawa T. Fukuda, G. Boulon, K. Kato, E. Hanamura, J. Cryst. Growth 287, 313 (2006).

[5] K. Izumi, S. Miyazaki, S. Yoshida, T. Mizokawa, E. Hanamura, Phys. Rev. B 76, 075111 (2007).

[6] A. Jouini, H. Sato, A. Yoshikawa, T. Fukuda, J. Mater. Res. 21, 2337 (2006).

[7] T. Sato, M. Shirai, K. Tanaka, Y. Kawabe, E. Hanamura, J. Lumin. 114, 155 (2005).

[8] J.H. Lim, B.N. Kim, Y. Kim, S. Kang, R.J. Xie, I.S. Chong, K. Morita, H. Yoshida, K. Hiraga, Appl. Phys. Lett. 102, 031104 (2013).

[9] A. Jouini, A. Yoshikawa, A. Brenier, T. Fukuda, G. Boulon, Phys. Status Solidi C 4, 1380 (2007).

[10] Yu.G. Kazarinov, V.T. Gritsyna, Yu.V. Sidorenko, Funct. Mater. 11, 131 (2004).

[11] E. Hanamura, Y. Kawabe, H. Takashima, T. Sato, A. Tomita, J. Nonlin. Opt. Phys. Mater. 12, 467 (2003).

[12] D.M. Levin, V.P. Gerasimov, F.Kh. Gusseinov, J. Appl. Spectrosc. 69, 492 (2001).

[13] V.T. Gritsyna, Yu.G. Kazarinov, V.A. Kobyakov, K.E. Sickafus, Radiat. Eff. Def. Solids 157, 659 (2002).

[14] S.T. Murphy, C.A. Gilbert, R. Smith, T.E. Mitchell, R.W. Grimes, Philos. Mag. 90, 1297 (2010).

[15] P.D. Borges, J. Cott, F.G. Pinto, J. Tronto, I. Scolfaro, Mater. Res. Expr. 3, 076202 (2016).

[16] B. Li, S.L. Oliveira, S.C. Rand, J.A. Azurdia, T.R. Hinklin, J.C. Marchal, R.M. Laine, J. Appl. Phys. 101, 053534 (2007). 